\section{Synchronous sporadic gastrointestinal stromal tumors (GISTs) of the colon}
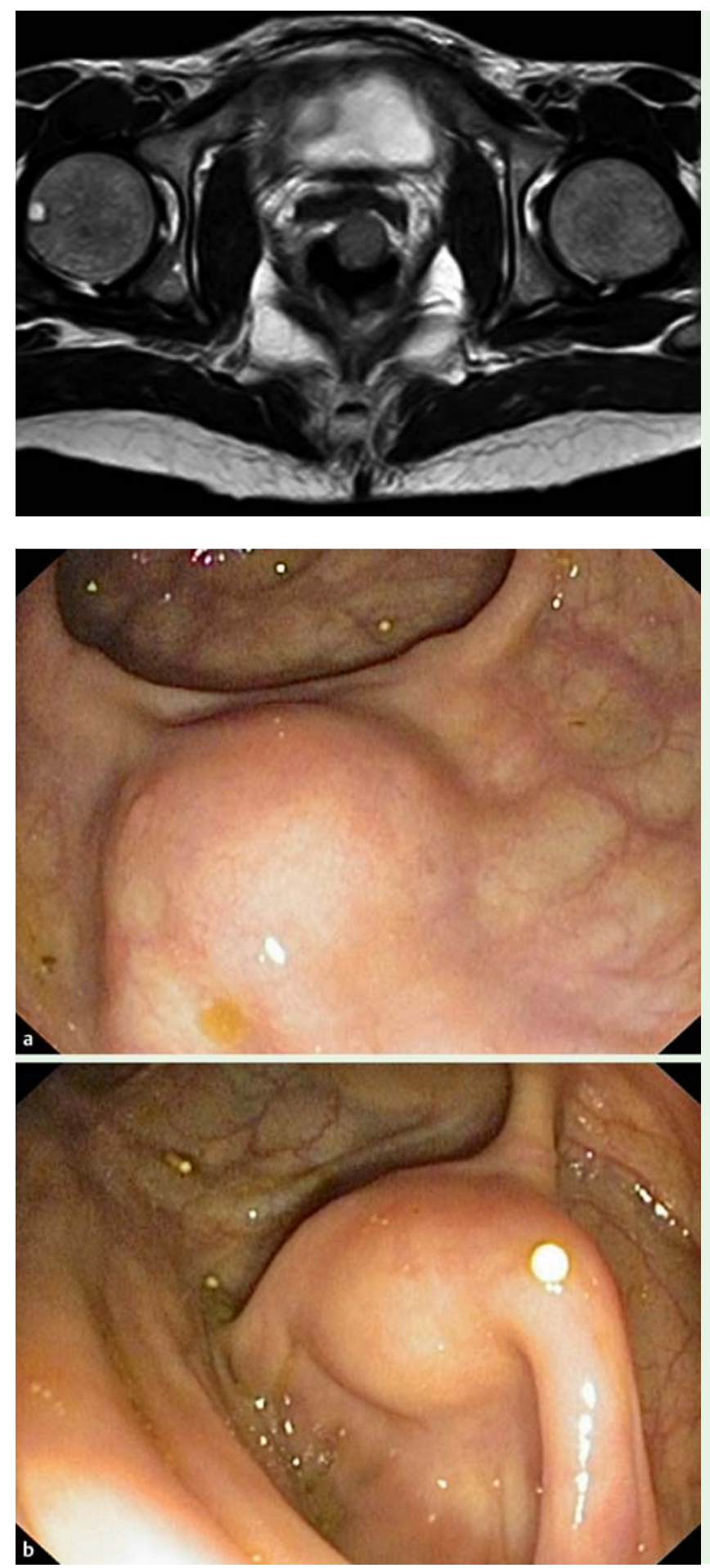

Gastrointestinal stromal tumors (GISTs) are rare mesenchymal tumors of the gastrointestinal tract originating from the cell of Cajal [1]. Up to 75\% of cases are driven by constitutional activation of the proto-oncogene $\mathrm{CKIT} ; 10 \%$ by a mutation
Fig. 1 Magnetic resonance imaging (MRI) scan showing a rectal gastrointestinal stromal tumor (GIST).

Fig. 2 Colonoscopy views showing: a a $2-\mathrm{cm}$ gastrointestinal stromal tumor (GIST) in the rectum; b a 2.5-cm GIST in the transverse colon.

of the gene encoding for the platelet-derived growth factor receptor (PDGFRA); $12 \%$ are wild-type and their pathogenesis has been related to mutations of the succinate dehydrogenase complex, BRAF or NF1 genes. Commonly GISTs arise as a solitary lesion; multiple spread is extremely rare and usually associated with familial GIST, type 1 neurofibromatosis (NF-1), or Carney's triad [2].

In July 2012, a 49-year-old healthy woman underwent a medical consultation after an episode of rectal bleeding. On examination she was found to have external hemorrhoids; digital rectal examination revealed an incidental finding of a $2-\mathrm{cm}$ nodule in the rectovaginal area, which was later confirmed by a pelvic magnetic resonance imaging (MRI) scan ( $\bullet$ Fig. 1 ). A colonoscopy showed a 2 -cm solid lesion at $6 \mathrm{~cm}$ from the anal margin (Fig. 2a) and a second solid lesion of $2.5 \mathrm{~cm}$ in the transverse colon ( Fig. 2b), both of which were covered with normal mucosa. An abdominal computed tomography (CT) scan confirmed the colonic lesion (๑ Fig.3).

The patient underwent resection of the transverse colon and enucleation of the rectal lesion. Histopathological examination of the colonic specimen showed a GIST of $1.5 \mathrm{~cm}$, with 1 mitosis per 50 high power fields (HPFs). Molecular analysis revealed a mutation in exon 11 of the cKIT gene. The rectal lesion was a GIST of $2 \mathrm{~cm}$ with 2 mitoses per 50 HPFs and a mutation in exon 9 of cKIT. Both GISTs were low risk for recurrence so no adjuvant therapy was given. The patient was started on clinical and radiological follow-up and is free from disease more than 1 year after her surgery.

Multiple sporadic GISTs not related to familial or NF-1 syndromes are a rare but recognized clinical presentation of GISTs and can occur as synchronous or metachronous spread. After the first publication, which reported five cases of multiple sporadic GISTs [2], other groups reported their experience, which showed the interest of experts in gaining a better understanding of the pathogenesis and clinical outcome of this peculiar presentation [3-6]. Synchronous second tumors have been reported in $13 \%$ of patient with GISTs and consideration of a differential diagnosis is mandatory for a correct therapeutic approach [7]. A molecular analysis is needed to distinguish between multiple primary GISTs and multiple recurrence or metastatic GISTs [7,8]. To our knowledge this is the first report of sporadic synchronous GISTs originating in the colorectal tract.

\section{Endoscopy_UCTN_Code_CCL_1AD_2AC}

Competing interests: None 


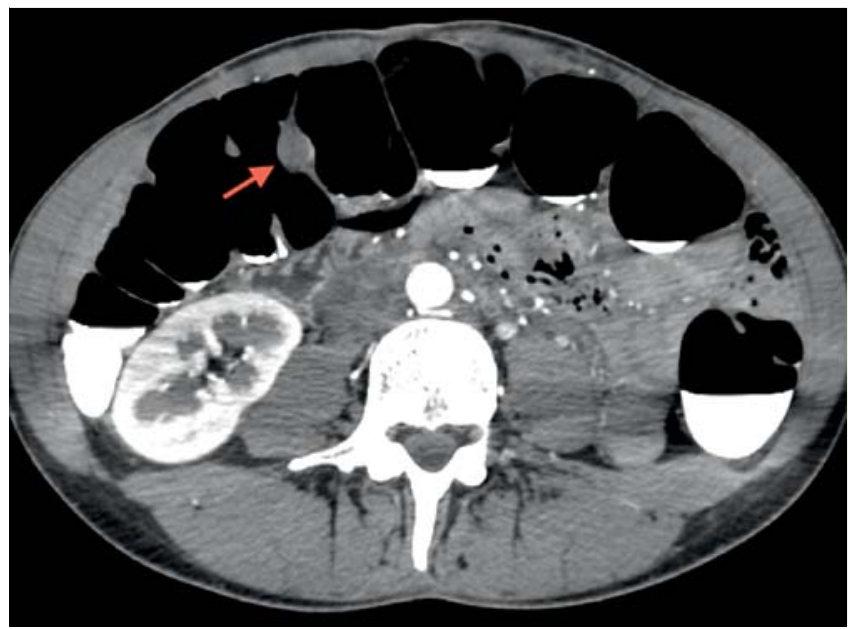

Fig. 3 Computed tomography (CT) scan showing the gastrointestinal stromal tumor (GIST) in the transverse colon (red arrow).

Fabio Accarpio ${ }^{1}$, Linda Cerbone ${ }^{2}$, Simone Sibio ${ }^{1}$, Marialuisa Framarino ${ }^{3}$, Daniele Biacchi ${ }^{1}$, Francesco Borrini ${ }^{4}$, Maurizio Cardi ${ }^{1}$, Sabrina Rossi ${ }^{5}$, Luisa Toffolatti ${ }^{5}$, Franco lafrate ${ }^{6}$, Andrea Laghi ${ }^{6}$, Angelo Di Giorgio ${ }^{1}$, Paolo Sammartino ${ }^{1}$

${ }^{1}$ Department of Surgery, P. Valdoni, Sapienza University of Rome, Umberto I Hospital, Rome, Italy

2 Department of Medical Oncology, San Camillo-Forlanini Hospital, Rome, Italy

${ }^{3}$ Department of Gynecology and Obstetrics, Sapienza University of Rome, Azienda Policlinico, Umberto I Hospital, Rome, Italy

${ }^{4}$ UOC of Pathology, Sandro Pertini Hospital, Rome, Italy
${ }^{5}$ Department of Pathology, Treviso General Hospital, Treviso, Italy

${ }^{6}$ Department of Radiology, Sapienza University of Rome, Umberto I Hospital, Rome, Italy

1 Badalamenti G, Rodolico V, Fulfaro $F$ et al. Gastrointestinal stromal tumors (GISTs): focus on histopathological diagnosis and biomolecular features. Ann Oncol 2007; 18 (Suppl. 06): 136-140

2 Kang DY, Park CK, Choi JS et al. Multiple gastrointestinal stromal tumors: clinicopathologic and genetic analysis of 12 patients. Am J Surg Pathol 2007; 31: 224-232

3 Haller F, Schulten HJ, Armbrust T et al. Multicentric sporadic gastrointestinal stromal tumors (GISTs) of the stomach with distinct clonal origin: differential diagnosis to familial and syndromal GIST variants and perito-

\section{References}

neal metastasis. Am J Surg Pathol 2007; 31: 933-937

4 Nakayama Y, Kadowaki K, Higure A et al. Synchronous sporadic gastrointestinal stromal tumors in the stomach and jejunum: report of a case. Case Rep Gastroenterol 2013; 7: $69-74$

$5 \mathrm{Xu}$ C, Liu YL, Yu HY et al. Synchronous and metachronous multiple gastrointestinal stromal tumors. Histol Histopathol 2012; 27: $225-234$

6 Samaras VD, Foukas PG, Triantafyllou K et al. Synchronous well differentiated neuroendocrine tumour and gastrointestinal stromal tumour of the stomach: a case report. BMC Gastroenterol 2011; 11: 27

7 Seshadri RRA, Singh SS, Ratnagiri R. Synchronous jejunal gastrointestinal stromal tumor and primary adenocarcinoma of the colon. Indian J Surg 2012; 74: 196-198

8 Gasparotto D, Rossi S, Bearzi I et al. Multiple primary sporadic gastrointestinal stromal tumors in the adult: an underestimated entity. Clin Cancer Res 2008; 14: 5715-5721

Bibliography

DOI http://dx.doi.org/ 10.1055/s-0034-1365443 Endoscopy 2014; 46: E252-E253 (c) Georg Thieme Verlag KG Stuttgart · New York ISSN 0013-726X

\section{Corresponding author}

\section{Linda Cerbone, MD}

Department of Medical Oncology, San Camillo-Forlanini Hospital

Circonvallazione Gianicolense 87 00151 Rome

Italy

Fax: +39-65-8704317

cerbone.linda@gmail.com 